\title{
Entrenamiento, Prueba y Predicción de la Solubilidad del Dióxido de Azufre en Líquidos lónicos utilizando Redes Neuronales Artificiales
}

\author{
Claudio A. Faúndez ${ }^{(1) *}$ y Richard A. Campusano ${ }^{(2,3)}$ \\ 1) Universidad de Concepción, Depto. de Física, Casilla 160-C, Concepción-Chile \\ (e-mail: claudiofaundez@udec.cl) \\ 2) Universidad de La Serena, Dpto. de Física y Astronomía, Casilla 554, La Serena-Chile \\ (e-mail: rcampusano27@gmail.com) \\ 3) Centro de Información Tecnológica, calle Monseñor Subercaseaux 667, La Serena-Chile \\ ${ }^{*}$ Autor a quien debe ser dirigida la correspondencia
}

Recibido Feb. 6, 2018; Aceptado Mar. 5, 2018; Versión final Mar. 20, 2018, Publicado Jun. 2018

\begin{abstract}
Resumen
Se analiza la capacidad de modelos de redes neuronales artificiales (RNA) para la predicción de la solubilidad de gases en líquidos iónicos (LIs). Se usan datos disponibles de solubilidad de dióxido de azufre $\left(\mathrm{SO}_{2}\right)$ en ocho líquidos iónicos, mezclas atractivas para procesos relacionados con la prevención de la contaminación ambiental, como, por ejemplo, el secuestro por absorción de $\mathrm{SO}_{2}$. Un total de 155 datos de solubilidad (P-T$\mathrm{x}$ ) para estos sistemas se tomaron de la literatura (131 datos para el entrenamiento, 16 para prueba y 8 para predicción). La variable dependiente es la solubilidad y las variables independientes son la temperatura ( $\mathrm{T})$ y la presión $(\mathrm{P})$, además de propiedades que identifican a cada líquido iónico, tales como temperatura crítica $\left(T_{c}\right)$, la presión crítica $\left(P_{c}\right)$, el índice de conectividad másico $(\lambda)$, el factor acéntrico $(\omega)$, la masa del catión $\left(\mathrm{M}^{+}\right)$ y la masa del anión (M-). Se analizaron tres casos, combinando estas variables independientes. El estudio muestra que el modelo de red neuronal propuesto $(2,6,1)$, con cinco variables en la capa de entrada $\left(T, P, T_{c}\right.$, $P_{c}$, y $\left.\omega\right)$, es la arquitectura adecuada en este estudio.
\end{abstract}

\section{Training, Test and Prediction of Solubility of Sulphur Dioxide in lonic Liquids Using Artificial Neural Networks}

\begin{abstract}
The capacity of artificial neural network models (ANN) to correlate and predict the solubility of gases in ionic liquids (ILs) is analyzed. Solubility data of sulfur dioxide $\left(\mathrm{SO}_{2}\right)$ in eight ionic liquids are considered. A total of 155 data points of solubility (pressure, temperature, and mole fraction; $T, P, x$ ) for these systems were taken from the literature. Of these total, 131 data were used for training the network, 16 for testing and 8 for prediction. The dependent variable (target variable) is the solubility and the independent variables are the temperature and pressure. Also some ionic liquid properties were employed as independent variables to distinguish between different ionic liquids: the critical temperature $\left(T_{c}\right)$, the critical pressure $\left(P_{c}\right)$, the mass connectivity index $(\lambda)$, the acentric factor $(\omega)$, the cation mass $\left(\mathrm{M}^{+}\right)$and the anion mass $\left(\mathrm{M}^{-}\right)$. Three cases were considered for analysis, modifying in each case the ionic liquid properties. The study shows that the proposed neural model $(2,6,1)$ with five variables in the inner layer $\left(T, P, T_{c}, P_{c}\right.$, and $\left.\omega\right)$, is the recommended architecture for this study.
\end{abstract}

Keywords: $\mathrm{SO}_{2}$ solubility; ionic liquids; gas-Iqiuid equilibrium; artificial neural networks 


\section{INTRODUCCIÓN}

El uso del dióxido de azufre $\left(\mathrm{SO}_{2}\right)$ se ha extendido ampliamente, tomando relevancia como materia prima en la industria de la celulosa y el papel, fabricación de textiles y procesado de alimentos. Estudios sobre el dióxido de azufre son de especial importancia debido a que este compuesto genera grandes problemas en la salud humana, contamina el ambiente y aportan negativamente al calentamiento global (Tiang et al., 2014). Muchos procesos industriales tienen como objetivo eliminar estos gases ácidos de las corrientes de gases de combustión (Pacala y Socolow, 2004). La absorción de $\mathrm{SO}_{2}$ por solventes orgánicos, tales como los líquidos iónicos (LIs) es uno de los procedimientos de captura comúnmente utilizados (Tiang et al., 2014). Hoy en día existen miles de sustancias considerablemente tóxicas, entre ellas neurotóxicas, en las cuales millones de trabajadores y personas en general se ven expuestos a sus consecuencias (Sánchez et al., 2014). La industria pretende diseñar líquidos iónicos en el área farmacéutica y de hidrometalurgia, áreas exploradas desde hace sólo un par de años atrás con LIs. El uso de líquidos iónicos como solventes industriales representa sin duda un avance en la protección del medio ambiente y de las personas.

Los líquidos iónicos (LIs), sustancias formadas solo por iones y usualmente en estado líquido a temperatura ambiente se caracterizan en forma genérica por su baja inflamabilidad, alta estabilidad térmica, baja o muy baja presión de vapor, temperatura de fusión por debajo de los $100^{\circ} \mathrm{C}$ y reciclabilidad. Por estas características se les ha identificado como solventes verdes (Hekayati et al., 2015). El nombre acuñado hace un tiempo como los "solventes del futuro" parece estar imponiéndose y nuevos estudios aparecen día a día en la literatura científica (Romero, 2008; Shamshina et al., 2015). Mezclas de gases con líquidos iónicos han encontrado cada vez mayores aplicaciones en la ingeniería química (Park et al., 2014, Lei et al., 2014). En particular, el estudio de la solubilidad del $\mathrm{SO}_{2}$ en LIs es una información relevante, para los procesos de separación del $\mathrm{SO}_{2}$ usando LIs como disolventes (Anderson et al., 2006). En la literatura se han reportado estudios sobre la captura del $\mathrm{SO}_{2}$ por Lls cuyos resultados han recibido especial atención (Anderson et al, 2006; Yokozeki y Shiflett, 2009; Shiflett y Yokozeki, 2010; Jin et al., 2011; Lei et al., 2014). Este trabajo aporta conocimiento para el tratamiento de datos de solubilidad en líquidos iónicos que puedan servir para la mejora del diseño y optimización de procesos.

Diferentes mezclas de gases y líquidos iónicos se han estudiado en la literatura usando varios modelos termodinámicos, principalmente ecuaciones de estado (EdE) (Faúndez et al., 2013; Valderrama et al., 2017a). La aplicación de una ecuación de estado a mezclas requiere conocer las propiedades de los componentes de la mezcla y el uso de reglas de mezcla. La precisión en la correlación de equilibrio líquido-vapor obtenida por el método de la EdE depende principalmente de la regla de mezcla y de la regla de combinación empleada para representar la dependencia de los parámetros de la EdE con la concentración (Valderrama, 2003). Usualmente, las reglas de mezcla y de combinación incluyen parámetros de interacción binarios para obtener correlaciones y predicciones más precisas. Estos parámetros se obtienen ajustando datos experimentales del equilibrio entre fases, mediante un proceso de optimización, que puede ser complejo. En algunos casos la predicción no es lo suficientemente precisa y las aplicaciones a procesos reales se vuelven difíciles de implementar (Karimi y Yousefi, 2007; Ghanadzadeh et al., 2008). De aquí que el desarrollo de métodos de estimación alternativos, como las RNA, ha demostrado ser exitosos para estimar datos de equilibrio de interés en el área de la ingeniería química (Nguyen et al., 2007; Eslamimanesh et al., 2011). En este trabajo se han seleccionado para estudio mezclas binarias de $\mathrm{SO}_{2}$ en 8 líquidos iónicos.

Las redes neuronales artificiales (RNA) es un método computacional de resolución de problemas (humanos, numéricos, mixtos, etc.) basado por similitud de sistemas biológicos compuestos por células nerviosas muy simples pero numerosas que trabajan masivamente en paralelo, que tienen la capacidad de aprender. El aprendizaje de la RNA; puede ser por capacitación, mediante estímulo, o por aprendizaje de refuerzo, no reduciéndose a la formulación de un algoritmo. En una RNA la relación entre variables dependientes (en este estudio la solubilidad) y otras independientes (las variables independientes temperatura $(T)$, presión $(P)$, temperatura crítica $\left(T_{c}\right)$, presión crítica $\left(P_{c}\right)$, índice de conectividad másico $(\lambda)$, factor acéntrico $(\omega)$, masa del catión $\left(\mathrm{M}^{+}\right)$y masa del anión $\left(\mathrm{M}^{-}\right)$, se relacionan mediante aprendizaje, de tal forma de imitar la manera de funcionar del sistema nervioso de los animales (Bose y Liang, 1996). La relación entre las variables dependientes y las variables independientes se establece entrenando la red, la que aprende a relacionar las variables. Esta característica de relacionar las variables mediante el aprendizaje es el elemento de su popularidad y de sus numerosas aplicaciones que se ven en la literatura en diferentes áreas de las ciencias y de la ingeniería. Descripciones detalladas de las RNA han sido presentadas en la literatura, en libros, artículos y monografías, por lo que no es necesario repetirlo aquí. El libro de Kriesel (2005) es una buena fuente para documentarse sobre RNA y sus aplicaciones. En los últimos años, las RNA han sido aplicadas para modelar diversas propiedades termodinámicas de mezclas que contienen líquidos iónicos (Nami y Deyhimi 2011, Álvarez y Saldaña, 2012). Modelos para correlacionar datos de solubilidad y de constantes de Henry usando RNA también han sido presentados por algunos autores (Eslamimanesh et al., 2011; Safamirzaei y Modarres, 2012; Lashkarbolooki et al., 2013; Faúndez et al., 2016). 
En este trabajo, se hace notar que una arquitectura con pesos y sesgos superior al número de datos disponibles, como la reportada en el trabajo de Bahmani et al., (2015) al utilizar una capa oculta con 27 neuronas, es inapropiado (Karsoliya, 2012). Tambien se realizó una tercera etapa de predicción, que según un estudio de Campusano (2017) y Valderrama et. al., (2017), listan trabajos, que presentan sólo entrenamiento y otros entrenamiento y prueba, sin la tercera etapa de predicción. Estos son los aportes más relevantes del presente estudio, es decir, cantidad de neuronas en función de la cantidad de datos y tercera etapa de predicción.

\section{METODOLOGÍA}

En este estudio se ha analizado la solubilidad del $\mathrm{SO}_{2}$ en varios líquidos iónicos: [Bmim][PF 6$]$, [Bmim][BF $]$, $[\mathrm{Bmim}][\mathrm{AC}],\left[\mathrm{Bmim}^{2}\left[\mathrm{MeSO}_{4}\right],\left[\mathrm{Bmim}^{2}\left[\mathrm{TF}_{2} \mathrm{~N}\right],[\mathrm{Emim}]\left[\mathrm{BF}_{4}\right],[\mathrm{Hmim}]\left[\mathrm{BF}_{4}\right]\right.\right.$ y $[\mathrm{Hmim}]\left[\mathrm{TF}_{2} \mathrm{~N}\right]$. Los intervalos de temperatura varían desde $283 \mathrm{~K}$ hasta $348 \mathrm{~K}$, los intervalos de presión varían desde 0.052 bares hasta 3.004 bares y los intervalos de solubilidad del $\mathrm{SO}_{2}$ en los líquidos iónicos desde 0.033 hasta 0.891 . El estudio incluye 17 isotermas con un total de 155 datos experimentales $(P, T, x)$. En la Tabla 1 se muestran las temperaturas críticas $\left(T_{c}\right)$, las presiones críticas $\left(P_{c}\right)$, el factor acéntrico $(\omega)$, la masa del anión $\left(M^{+}\right)$, la masa del catión $\left(M^{-}\right)$ y el índice de conectividad másico $(\lambda)$ de todos los líquidos iónicos considerados en este estudio. Las propiedades críticas $\left(T_{c}, P_{c}\right)$ y el factor acéntrico $(\omega)$ de los líquidos iónicos fueron obtenidos del trabajo de Valderrama et al. (2012), mientras que los valores para la masa del anión, la masa del catión y el índice de conectividad másico del artículo de Valderrama et al., (2017b). La Tabla 2 muestra los intervalos de temperatura, presión, y solubilidad de los datos utilizados en este trabajo y la literatura de donde fueron tomados los datos experimentales.

Las variables de entrenamiento son la temperatura y la presión de estos sistemas binarios $(T, P)$ siendo la variable dependiente (variable objetivo) la solubilidad del $\mathrm{SO}_{2}$ en el líquido iónico. Para distinguir entre los líquidos iónicos en las mezclas estudiadas, se utilizaron tres propiedades características de los líquidos iónicos y se analizaron tres casos: 1) propiedades características: la temperatura y presión del sistema, la temperatura crítica, la presión crítica y el índice de conectividad másico ( $\left.T, P, T_{c}, P_{c}, \lambda\right)$; 2) propiedades características: la temperatura y presión del sistema, la temperatura crítica, la presión crítica y el factor acéntrico ( $\left.T, P, T_{c}, P_{c}, \omega\right)$; y 3 ) propiedades características: la temperatura y presión del sistema, la masa del anión, la masa del catión y el índice de conectividad másico ( $\left.T, P, M^{+}, M^{-}, \lambda\right)$. Por lo tanto, los parámetros de entrada para cada caso estudiado con la cantidad de variables independientes. La variable de salida es igual a 1 (la solubilidad del $\mathrm{SO}_{2}$ en el LI). La RNA fue entrenada con 131 datos, siendo 16 datos para prueba y 8 para predicción. Los datos para la prueba se seleccionaron de tal forma que no fueran ni el mínimo ni el máximo de la solubilidad para cada sistema y para la predicción, un dato dentro del intervalo entre dichos extremos.

Tabla 1: Propiedades de los LI utilizados en este trabajo para los casos estudiados.

\begin{tabular}{|l|c|c|c|c|c|c|}
\hline \multirow{2}{*}{\multicolumn{1}{c|}{$\begin{array}{c}\text { Mezcla } \\
\mathrm{SO}_{2}+\end{array}$}} & \multicolumn{6}{c|}{ Propiedades } \\
\cline { 2 - 7 } & $T_{c} / \mathrm{K}$ & $P_{c} /$ bar & $\omega$ & $M+$ & $M-$ & $\lambda$ \\
\hline$[$ Bmim][PF6] & 719.4 & 17.3 & 0.7917 & 139.22 & 144.922 & 2.040 \\
\hline$[$ Bmim][BF4] & 643.2 & 20.4 & 0.8877 & 139.22 & 86.803 & 0.5773 \\
\hline$[$ Bmim][AC] & 847.3 & 24.4 & 0.6681 & 139.22 & 59.046 & 0.6656 \\
\hline$[$ Bmim][MeSO $]$ & 1081.6 & 36.1 & 0.4111 & 139.22 & 111.1 & 0.6130 \\
\hline$[$ Bmim] $[$ TF2N] & 1269.9 & 27.65 & 0.3004 & 139.22 & 280.15 & 1.0952 \\
\hline$[$ Emim][BF4] & 596.2 & 23.59 & 0.8087 & 111.17 & 86.803 & 0.2921 \\
\hline$[$ Hmim][BF4] & 690.0 & 17.94 & 0.9625 & 167.28 & 86.803 & 0.8625 \\
\hline$[$ Hmim][TF2N] & 1298.8 & 23.9 & 0.3893 & 167.28 & 280.15 & 1.0952 \\
\hline
\end{tabular}

En este trabajo se utilizó el software Matlab y se escribieron los siguientes archivos para Matlab: a) un archivo Excel que contiene las variables independientes: temperatura y presión de la mezcla binaria y las propiedades características, que a modo de ejemplo para el caso 1 son ( $\left.T, P, T_{c}, P_{c}, \lambda\right)$; de cada uno de los líquidos iónicos; b) un archivo de Excel que contiene la variable dependiente, que es la solubilidad $\mathrm{x}_{1}$ del $\mathrm{SO}_{2}$ en el líquido iónico; y c) un código de Matlab que consta de tres partes: una sección de entrenamiento, una sección de prueba y una sección de predicción. En la sección de entrenamiento, el programa lee los datos de entrada (los dos archivos de Excel) define la arquitectura, entrena la red definida, genera las matrices de peso y sesgo, y almacena dichos datos para la prueba. En la sección de prueba y predicción, el programa lee las matrices de peso y sesgo, el archivo de Excel que contiene las variables para las cuales desea predecir la solubilidad y almacena los resultados en un archivo de salida. Un ejemplo detallado, del tipo de programa utilizado en este estudio se encuentra en la literatura (Faúndez et al., 2016). 
Tabla 2: Intervalos de solubilidad, temperatura y presión considerados en este trabajo

\begin{tabular}{|c|c|c|c|c|c|}
\hline Mezcla: $\mathrm{SO}_{2}+\mathrm{LI}$ & Referencia & $N$ & $T / K$ & Intervalo de $x_{1}$ & Intervalo de P/Bar \\
\hline$[\mathrm{Bmim}]\left[\mathrm{PF}_{6}\right]$ & Lei et al., 2014 & 5 & 298 & $0.191-0.693$ & $0.197-1.976$ \\
\hline$[\mathrm{Bmim}]\left[\mathrm{BF}_{4}\right]$ & Lei et al., 2014 & 7 & 298 & $0.187-0.697$ & $0.208-2.011$ \\
\hline \multirow[t]{4}{*}[\mathrm{Bmim}]{$[\mathrm{AC}]$} & \multirow[t]{4}{*}{ Shiflett y Yokoseki, 2010} & 6 & 283 & $0.484-0.863$ & $0.103-1.758$ \\
\hline & & 11 & 298 & $0.332-0.861$ & $0.052-3.017$ \\
\hline & & 11 & 323 & $0.181-0.679$ & $0.054-2.999$ \\
\hline & & 11 & 348 & $0.110-0.560$ & $0.053-3.003$ \\
\hline \multirow[t]{4}{*}[\mathrm{Bmim}]{$\left[\mathrm{MeSO}_{4}\right]$} & \multirow[t]{4}{*}{ Shiflett y Yokoseki, 2010} & 9 & 283 & $0.328-0.891$ & $0.053-1.754$ \\
\hline & & 11 & 298 & $0.332-0.861$ & $0.052-1.754$ \\
\hline & & 10 & 323 & $0.144-0.714$ & $0.052-3.017$ \\
\hline & & 11 & 348 & $0.033-0.568$ & $0.103-3.000$ \\
\hline$[\mathrm{Bmim}]\left[\mathrm{TF}_{2} \mathrm{~N}\right]$ & Lei et al., 2014 & 10 & 298 & $0.158-0.814$ & $0.173-2.476$ \\
\hline$[\mathrm{Emim}]\left[\mathrm{BF}_{4}\right]$ & Lei et al., 2014 & 11 & 298 & $0.173-0.732$ & $0.168-2.055$ \\
\hline$[\mathrm{Hmim}]\left[\mathrm{BF}_{4}\right]$ & Lei et al., 2014 & 11 & 298 & $0.179-0.760$ & $0.145-2.048$ \\
\hline \multirow[t]{4}{*}[\mathrm{Hmim}]{$\left[\mathrm{TF}_{2} \mathrm{~N}\right]$} & \multirow[t]{4}{*}{ Yokoseki y Shiflett, 2010} & 7 & 283 & $0.171-0.875$ & $0.107-1.750$ \\
\hline & & 8 & 298 & $0.124-0.881$ & $0.109-3.002$ \\
\hline & & 8 & 323 & $0.049-0.639$ & $0.109-3.002$ \\
\hline & & 7 & 348 & $0.124-0.457$ & $0.504-3.003$ \\
\hline
\end{tabular}

La arquitectura normalmente utilizada en este tipo de aplicaciones implica una red neuronal de propagación hacia atrás, que contiene la capa de entrada (variables independientes), dos capas ocultas, y una capa de salida (una variable dependiente). Campusano (2017) y Valderrama et al., (2017) en un estudio de temperatura de transición vítrea de líquidos iónicos, utilizaron la siguiente topología (o arquitectura) para la RNA, que ha sido utilizada en este estudio: (i) el número de iteraciones del orden de 1000; (ii) función de trasferencia tangente sigmoidal (tansig) para las capas ocultas y lineal para la capa de salida; (iii) error cuadrático medio (ECM) de los valores de la variable dependiente 10-6; (iv) alrededor del 10\% de los datos totales para la prueba y $10 \%$ para predicción, escogidos en forma aleatoria. El número de datos utilizados para entrena-miento debe ser al menos el doble del número de parámetros entregados por la red (pesos y sesgos). Los criterios anteriores, han demostrado ser confiables y válidos. El método de optimización utilizado es el LevenbergMarquardt, dado que, en general tiene una convergencia rápida, en el sentido que necesita menor cantidad de iteraciones para alcanzar el nivel especificado de error (Villada et al., 2014).

Se ha demostrado que esta arquitectura ha funcionado bien en otras aplicaciones sobre estimación de propiedades físicas y termodinámicas de fluidos (Valderrama et al., 2011; Faúndez et al., 2016). Como metodología de trabajo los valores de las variables independientes usadas para prueba y predicción deben estar dentro de los intervalos utilizados para el entrenamiento de la red (Tablas 1 y 2). Esto porque las redes neuronales artificiales son, en general, buenas herramientas para la interpolación pero no para la extrapolación (Valderrama, et al., 2017b).

\section{RESULTADOS Y DISCUSIÓN}

Se probaron varias arquitecturas de tres capas (más la de entrada) para seleccionar la topología (arquitectura) más simple de la RNA que dé resultados aceptables. El número óptimo de neuronas se determinó por ensayo y error. La simplicidad de la arquitectura y la exactitud de los resultados fueron los requisitos impuestos para encontrar la arquitectura óptima. La exactitud de la red final elegida se verificó determinando la desviación relativa promedio $\left(\% \Delta \mathrm{x}_{1}\right)$, la desviación absoluta relativa promedio ( $\left.\% \Delta \mathrm{x}_{1} \mid\right)$ y la máxima desviación relativa absoluta (máx. $\left|\% \Delta \mathrm{x}_{1}\right|$ ) de los valores calculados de $\mathrm{x}_{1}$ después del entrenamiento, de la prueba y de la predicción y los datos de la literatura. Estas desviaciones, para un conjunto de $\mathrm{N}$ puntos, se definen en las ecuaciones (1) a (3).

$$
\begin{aligned}
& \% \Delta x=\frac{100}{N} \sum \frac{x_{1}^{c a l}-x_{1}^{\exp }}{x_{1}^{\exp }} \\
& |\% \Delta x|=\frac{100}{N} \sum \frac{\left|x_{i}^{c a l}-x_{i}^{\exp }\right|}{x_{i}^{\exp }}
\end{aligned}
$$




$$
\left|\% \Delta x_{i}\right|=\max \left|\frac{x_{i}^{c a l}-x_{i}^{\exp }}{x_{i}^{\exp }}\right|
$$

Las ecuaciones 1 a la 3, ha demostrado ser excelentes parámetros para determinar la bondad de un modelo, y son los parámetros estadísticos usados para decidir sobre la red óptima. La red final utilizada tiene dos capas ocultas de 2 y 6 neuronas cada una. La capa de entrada es de 5 y la de salida de una neurona. La arquitectura optima es entonces $(2,6,1)$ o como se usa en otra nomenclatura que considera que matriz de entrada constituye una capa, es $(5,2,6,1)$. Durante el entrenamiento, las desviaciones individuales absolutas entre los valores correlacionados y experimentales del $\mathrm{SO}_{2}$ en el líquido iónico se detallan en la tabla 3 . Para cada fase de entrenamiento, la red se ejecutó 50 veces. Cada ejecución corresponde a un proceso de entrenamiento, vale decir, cada arquitectura se entrenó 50 veces. Realizarlo más veces no es necesario, por cuanto los parámetros estadísticos de las ecuaciones 1 a la 3, no cambian. Debe siempre haber entrenamiento, prueba y predicción y es necesario que los datos sean excluyentes (Valderrama et al., 2017b). En la Tabla 3, se muestran tres casos de las cincuenta ejecuciones, para los tres casos de estudio. De las cincuenta ejecuciones, se escogieron 3 que tuviesen las desviaciones más bajas (modelo a, b y c) tanto para entrenamiento, prueba y predicción.

Tabla 3: casos de estudio analizados en este trabajo.

\begin{tabular}{|c|c|c|c|c|c|c|c|c|c|c|}
\hline \multirow{2}{*}{$\begin{array}{c}\text { Arquitectura: } \\
2,6,1 \\
\end{array}$} & \multirow{3}{*}{$\begin{array}{l}\frac{0}{10} \\
\frac{0}{2} \\
\frac{0}{2}\end{array}$} & \multirow{2}{*}{\multicolumn{3}{|c|}{$\begin{array}{c}\text { Entrenamiento } \\
\text { (131 datos) }\end{array}$}} & \multirow{2}{*}{\multicolumn{3}{|c|}{$\begin{array}{c}\text { Prueba } \\
\text { (16 datos datos) }\end{array}$}} & \multirow{2}{*}{\multicolumn{3}{|c|}{$\begin{array}{l}\text { Predicción } \\
\text { (8 datos) }\end{array}$}} \\
\hline & & & & & & & & & & \\
\hline $\begin{array}{c}\text { Variables } \\
\text { Independientes }\end{array}$ & & $\% \Delta x$ & $|\% \Delta x|$ & $\begin{array}{l}\text { máx. } \\
|\% \Delta x|\end{array}$ & $\% \Delta x$ & $|\% \Delta x|$ & $\begin{array}{l}\text { máx. } \\
|\% \Delta x|\end{array}$ & $|\% \Delta x|$ & $\% \Delta x$ & $\begin{array}{l}\text { máx. } \\
|\% \Delta x|\end{array}$ \\
\hline \multirow{3}{*}{$\begin{array}{c}\text { Caso 1) } \\
\mathrm{T}, \mathrm{P}, \mathrm{T}_{\mathrm{c}}, \mathrm{P}_{\mathrm{c}}, \lambda\end{array}$} & a) & 0.8 & 7.2 & 57.5 & -1.3 & 5.1 & 11.9 & 3.0 & 3.9 & 8.4 \\
\hline & b) & 1.0 & 7.0 & 31.9 & -0.2 & 6.9 & 16.7 & 1.2 & 5.3 & 15.0 \\
\hline & c) & 1.0 & 7.3 & 66.8 & -1.2 & 4.6 & 11.2 & 4.8 & 6.9 & 19.9 \\
\hline \multirow{3}{*}{$\begin{array}{c}\text { Caso 2) } \\
\mathrm{T}, \mathrm{P}, \mathrm{Tc}, \mathrm{Pc}, \omega\end{array}$} & a) & 1.0 & 6.6 & 60.2 & -1.1 & 4.3 & 11.8 & 2.2 & 6.7 & 18.5 \\
\hline & b) & 1.0 & 5.6 & 56.9 & -0.3 & 3.7 & 9.9 & 1.1 & 3.9 & 7.4 \\
\hline & c) & 0.9 & 7.2 & 44.6 & -1.6 & 5.5 & 12.8 & 0.5 & 4.0 & 11.2 \\
\hline \multirow{3}{*}{$\begin{array}{c}\text { Caso 3) } \\
\mathrm{T}, \mathrm{P}, \mathrm{M}_{+}, \mathrm{M}-, \lambda\end{array}$} & a) & 2.4 & 10.0 & 128.6 & 2.3 & 10.8 & 39.1 & 4.0 & 8.7 & 19.9 \\
\hline & b) & 3.0 & 11.7 & 143.1 & 1.2 & 15.5 & 49.0 & 4.4 & 10.3 & 29.6 \\
\hline & c) & 2.9 & 11.5 & 128.8 & 0.9 & 13.0 & 54.9 & 5.9 & 12.0 & 28.3 \\
\hline
\end{tabular}

Las variables más apropiadas del estudio realizado son el modelo a) del caso $1\left(T, P, T_{c}, P_{c}, \lambda\right)$ y el modelo b) del caso $2\left(T, P, T_{c}, P_{c}, \omega\right)$. El caso $3(T, P, M+, M-, \lambda)$ es descartando, por cuanto la RNA no tiene capacidad predictiva, y las desviaciones máximas de entrenamiento son sobre el $100 \%$, por lo que la red no fue entrena adecuadamente. La Figura 1, muestra la distribución de los errores para el proceso de entrenamiento considerando los tres casos de estudio. La Tabla 4, muestra los errores individuales para los casos escogidos en la etapa de predicción. La capacidad predictiva del modelo puede ser observada en la Figura 2. En la figura se muestran los datos experimentales y los calculados para prueba y prediccion para el modelo final propuesto (el modelo b del caso dos de la tabla 3). El mismo procedimiento realizado para la arquitectura $(2,6,1)$ con las variables $\left(T, P, T_{c}, P_{c}, \omega\right)$ se aplicó para la arquitecturas $(2,8,1)$ la que para la etapa de predicción da desviación media de $-0.8 \%$, desviación media absoluta de $3.1 \%$ y máxima de $4.8 \%$. Resultados similares se obtuvo con una arquitectura $(4,4,1)$ que para predicción da desviación media de $0.5 \%$, desviación media absoluta de $0.5 \%$ y máxima de $1.6 \%$. Estas arquitecturas que parecen también aceptables, tienen 8 y 10 parámetros más, respectivamente que la recomendada $(2,6,1)$.

Tabla 4: Errores individuales para predicción con los casos seleccionados en la tabla 3

\begin{tabular}{|l|c|c|c|c|c|c|c|c|}
\hline \multirow{2}{*}{$\mathrm{SO}_{2}+\mathrm{LI}$} & \multirow{2}{*}{$T(K)$} & Datos predicción & \multicolumn{3}{|c|}{ Modelo con $T, P, T_{c}, P_{c}, \lambda$} & \multicolumn{3}{c|}{ Modelo con $T, P, T_{c}, P_{c}, \omega$} \\
\cline { 3 - 8 } & & $\mathrm{x}$ exp. & $\mathrm{x}$ cal. & $\mid \% \Delta \mathrm{x}$ & $\% \Delta \mathrm{x}$ & $\mathrm{x}_{\text {cal. }}$ & $|\% \Delta \mathrm{x}|$ & $\% \Delta \mathrm{x}$ \\
\hline$[\mathrm{BMIM}][\mathrm{AC}]$ & 298 & 0.861 & 0.9234 & 7.3 & 7.3 & 0.8763 & 1.8 & 1.8 \\
\hline$[\mathrm{Bmim}]\left[\mathrm{MeSO}_{4}\right]$ & 323 & 0.638 & 0.6433 & 0.8 & 0.8 & 0.6397 & 0.3 & 0.3 \\
\hline$[\mathrm{Hmim}]\left[\mathrm{TF}_{2} \mathrm{~N}\right]$ & 323 & 0.513 & 0.5189 & 1.2 & 1.2 & 0.5486 & 6.9 & 6.9 \\
\hline$[\mathrm{Bmim}]\left[\mathrm{BF}_{4}\right]$ & 298 & 0.610 & 0.6613 & 8.4 & 8.4 & 0.6493 & 6.4 & 6.4 \\
\hline$[\mathrm{Emim}]\left[\mathrm{BF}_{4}\right]$ & 298 & 0.413 & 0.3982 & -3.7 & 3.7 & 0.3827 & -7.4 & 7.4 \\
\hline$[\mathrm{Hmim}]\left[\mathrm{BF}_{4}\right]$ & 298 & 0.506 & 0.5161 & 1.9 & 1.9 & 0.4933 & -2.6 & 2.6 \\
\hline$[\mathrm{Bmim}]\left[\mathrm{TF}_{2} \mathrm{~N}\right]$ & 298 & 0.747 & 0.7487 & 0.2 & 0.2 & 0.7634 & 2.2 & 2.2 \\
\hline$[\mathrm{Bmim}]\left[\mathrm{PF}_{6}\right]$ & 298 & 0.637 & 0.6862 & 7.7 & 7.7 & 0.8763 & 1.8 & 1.8 \\
\hline
\end{tabular}


Algunos modelos, para la estimación de la solubilidad del $\mathrm{SO}_{2}$ en $\mathrm{Lls}$ han sido presentados en la literatura. Yokoseki y Shiflett (2009) han medido experimentalmente solubilidades del $\mathrm{SO}_{2}$ en el líquido iónico [Hmim] $\left[\mathrm{TF}_{2} \mathrm{~N}\right]$ a temperaturas entre 283 y $348 \mathrm{~K}$. Los resultados experimentales están en acuerdo con los obtenidos con una EdE basada en el modelo de Redlich-Kwong. En otro trabajo, Yokoseki y Shiflett (2010), han mostrado que los datos experimentales del $\mathrm{SO}_{2}$ en el líquido iónico [Bmim][MeSO 4 , a temperaturas entre 283 y $348 \mathrm{~K}$. Los resultados experimentales se modelaron con una ecuación de estado cúbica tipo van der Waals. Bahmani et al., (2015) ha utilizado una RNA para estimar la solubilidad del $\mathrm{SO}_{2}$ en 8 LIs. Estos autores proponen una compleja RNA que tiene 27 neuronas en la capa oculta, número que resulta excesivo considerando la poca cantidad de datos experimentales (232 datos). La desviación absoluta promedio en predicción es de $2.5 \%$ y máximas desviaciones absolutas del $9.7 \%$ son encontradas en los sistemas $\mathrm{SO} 2+[\mathrm{BMIM}][\mathrm{AC}]$ y $\mathrm{SO}_{2}+$ [Bmim] $\left[\mathrm{MeSO}_{4}\right]$ a la temperatura de $348 \mathrm{~K}$.

Una contribución de este trabajo es hacer notar que una arquitectura con mayor número de neuronas, no implica mejores resultados para entrenamiento, prueba o predicción, como lo mostrado con la red $(4,4,1)$. Karsoliya (2012) señala: "Al aumentar el número de capas ocultas hasta tres capas, se puede mejorar la exactitud en los resultados, pero la complejidad de la red y el tiempo de entrenamiento pueda aumentar enormemente. Si la precisión es el criterio principal para el diseño de una red neuronal, las capas ocultas pueden ser aumentadas. Pero el incremento innecesario en las neuronas o capas puede causar un problema de sobreajuste o memorización. Por lo tanto, es esencial que antes de diseñar la red neuronal, se analicen muestras de los datos disponibles de modo que el número de capas y neuronas pueda ser supuesto en forma razonable", situación que se presenta en la excesiva arquitectura en Bahmani et al., (2015) para su cantidad de datos, los que además, no utilizan, al igual que varios autores (Campusano, 2017) un tercer conjunto de datos para predicción, no pudiéndose garantizar la capacidad predictiva del modelo. Seguir aumentando la arquitectura de la red, aunque se mejoren los errores, va en contra de lo establecido por Karsoliya (2012), Campusano (2017) y por Valderrama et al., (2017), que es lo indebidamente aplicado por Bahmani et al., (2015) al utilizar una capa oculta con 27 neuronas.
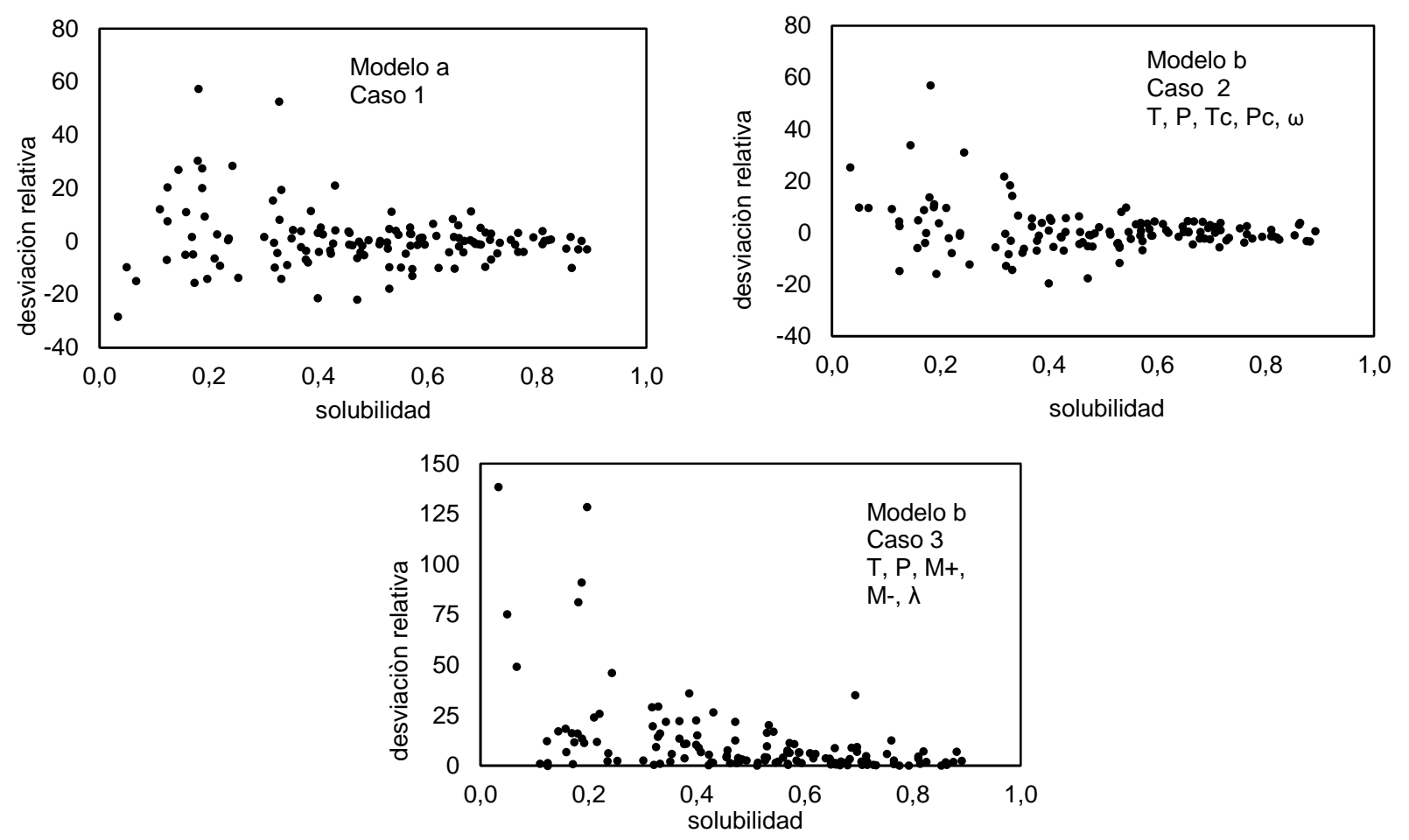

Fig. 1: Desviación relativa para el proceso de entrenamiento para cada caso escogido en la tabla 3

Un entrenamiento con desviaciones relativas sobre el $40 \%$, no implica que la red no tenga capacidad predictiva como se muestra en la tabla 3 , aun cuando hay máximas desviaciones relativas absolutas del orden del $20 \%$. Esto obedece a que la RNA, tiene como características capacidad de autorregulación, de generalización y tolerancia a los fallos. Kriesel (2005) señala "después de una capacitación exitosa, una red neuronal puede encontrar soluciones razonables para problemas similares de la misma clase que no fueron entrenados explícitamente. Esto, a su vez, da como resultado un alto grado de tolerancia a fallas frente a datos de entrada ruidosos. A pesar de un proceso de enteramiento no exitoso, con las desviaciones ya mencionadas, el proceso de prueba y de enteramiento, según el criterio del lector, pueden considerarse exitosos la prueba y la predicción, es decir: modelo a) caso 1 ; y modelo b) caso 2. 


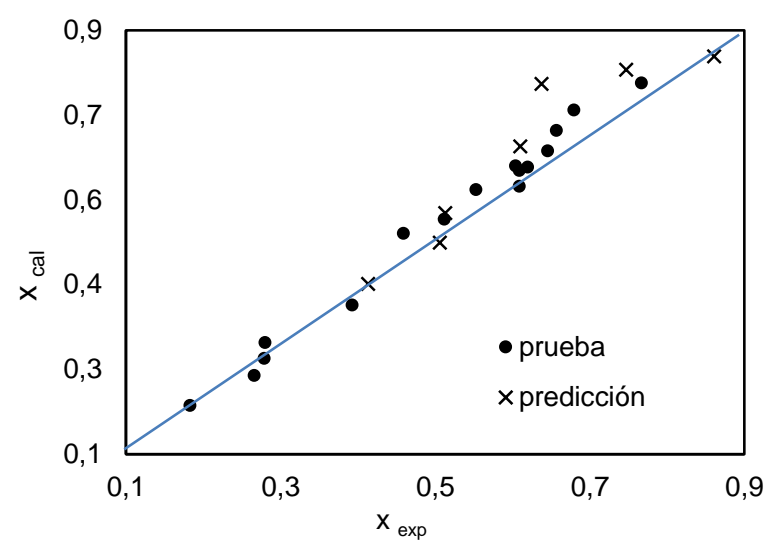

Fig. 2: datos calculados versus experimentales del Caso 2, modelo b en la tabla $3\left(T, P, T_{c}, P_{c}, \omega\right)$.

\section{CONCLUSIONES}

i) Se ha desarrollado un modelo de red neuronal artificial, basado en una simple arquitectura de dos capas ocultas, para entrenar, probar y predecir la solubilidad del $\mathrm{SO}_{2}$ en diferentes líquidos iónicos, encontrándose resultados aceptables.

ii) Se usó tres combinaciones de variables independientes, (T, $\left.P, T_{c}, P_{c}, \lambda\right) ;\left(T, P, T_{c}, P_{c}, \omega\right) ; y\left(T, P, M^{+}, M^{-}, \lambda\right)$. Los mejores resultados en prueba y predicción se dan con la combinación ( $\left.T, P, T_{c}, P_{c}, \omega\right)$.

iii) Los resultados con la red $(2,6,1)$ y las variables $\left(T, P, M^{+}, M^{-}, \omega\right)$ predicen la solubilidad con una desviación relativa absoluta promedio total de $3.7 \%$, siendo la máxima desviación individual de $7.4 \%$.

iv) Un aumento en el número de neuronas en la arquitectura de la red, no implica una mejora sustantiva en los resultados de entrenamiento, prueba y predicción.

v) Al trabajar con RNA, se necesitan tres conjuntos excluyentes de datos, entrenamiento, prueba y predicción. De no serlo, no se puede garantizar la capacidad predictiva del modelo.

\section{AGRADECIMIENTOS}

Los autores agradecen a la Comisión Nacional de Investigación Científica y Tecnológica (CONICYT) por su apoyo a través del Proyecto Fondecyt $N^{\circ} 1150802$ y agradecen el soporte computacional del Centro de Información Tecnológica de La Serena (Chile). CAF agradece al Departamento de Física de la Universidad de Concepción (Chile) por su apoyo durante la elaboración de este trabajo. RAC agradece a la Vicerrectoría de Investigación y Posgrado y al Departamento de Física y Astronomía, ambos de la Universidad de La Serena (Chile), por su apoyo durante la elaboración de este trabajo.

\section{REFERENCIAS}

Agami, N.; A. Atiya, M. Saleh, El-Shishiny. A Neural Network Based Dynamic Forecasting Model for Trend Impact Analysis. Technological Forecasting \& Social Change, 76, 952-962, (2009)

Alvarez, V.H.; M.D.A. Saldaña. Thermodynamic prediction of vapor-liquid equilibrium of supercritical $\mathrm{CO} 2$ or $\mathrm{CHF} 3+$ ionic liquids. J. Supercrit. Fluids, https://doi.org/10.1016/j.supflu.2012.02.011, 66, 29-35, (2012)

Anderson, J.L.; J.K. Dixon, E.J. Maginn, J.F. Brennecke. Measurement of SO2 solubility in ionic liquids, J. Phys. Chem. B 110 15059-15062, (2006)

Bahmani, A.R.; Fatemeh, S., Bahmani, M. Prediction of solubility of sulfur dioxide in ionic liquids using artificial neural network. Journal of Molecular Liquids, 211 395-400, (2015)

Campusano, R. Propiedades de Transición de Fases Líquido-Sólido en Líquidos lónicos. Tesis de Maestría en Ciencias Físicas, Departamento de Física y Astronomía, Facultad de Ciencias, Universidad de La Serena-Chile, Enero (2017)

Eslamimanesh, A.; F. Gharagheizi, A.H. Mohammadi, D. Richon. Artificial neural network modeling of solubility of supercritical $\mathrm{CO}_{2}$ in 24 commonly used ionic liquids, Chem. Eng. Sci. 66(13), 3039-3044, (2011)

Faúndez, C.; L.A. Barrientos, J.O. Valderrama. Modeling and thermodynamic consistency of solubility data of refrigerants in ionic liquids. Int. J. Refrig., 36 (8), 2242-2250, (2013)

Faúndez, C.; E.N. Fierro, J.O. Valderrama. Solubility of hydrogen sulfide in ionic liquids for gas removal processes using artificial neural networks. J. Environm.Chem. Eng., http://dx.doi.org/10.1016/j.jece.2015.11.008, 36,4(1), 211-218, (2016) 
Ghanadzadeh, H.; H. Ahmadifar. Estimation of (vapor-liquid) equilibrium of binary systems (tert-butanol+2-ethyl-1-hexanol) and (nbutanol+2-ethyl-1-hexanol) using an artificial neural network. J. Chem. Thermodyn., 40 1152, (2008)

Hekayati, J.; A. Roosta, J., Javanmardi. Thermodynamic Modeling of Refrigerant Solubility in lonic Liquids Using Original and e-Modified Sanchez-Lacombe Equation. Fluid Phase Equilib., 403, 14-22, (2015)

Jin, M.; Y. Hou,W. Wu, S. Ren, S. Tian, L. Xiao, Z. Lei. Solubilities and thermodynamic properties of SO2 in ionic liquids. J. Phys. Chem. B 115) 6585-6591, (2011)

Karimi, H.; F. Yousefi, Correlation of vapour liquid equilibria of binary mixtures using artificial neural networkChin. J. Chem. Eng. 15 765-771, (2007)

Karsoliya, S. Approximating Number of Hidden Layer Neurons in Multiple Hidden Layer BPNN Architecture. International Journal of Engineering Trends and Technology, ISSN: 2231-5381, 3 (6), 714-717, (2012)

Kriesel, D. A Brief Introduction of Neural Networks. (2005). https://goo.gl/qfFNSZ. Acceso: 23 sep. (2015)

Lashkarbolooki, M.; A.Z. Hezave, A. Babapoor. Correlation of density for binary mixtures of methanol+ionic liquids using back propagation artificial neural network. Korean J. Chem. Eng., 30(1), 213-220, (2013)

Lei, Z.; C. Dai, B. Chen. Gas solubility in ionic liquids. Chem. Rev. 114 1289-1326, (2014)

Nami, F.; F. Deyhimi. Prediction of activity coefficients at infinite dilution for organic solutes in ionic liquids by artificial neural network, J. Chem. Thermodyn., 43 22, (2011)

Nguyen. V.D.; R. R. Tan. Y. Brondial. T. Fuchino. Prediction of vapor-liquid equilibrium data for ternary systems using artificial neural networks. Fluid Phase Equilibria, 254, 188 - 197, (2007)

Pacala, S.; R. Socolow. Stabilization wedges: solving the climate problem for the next 50 years with current technologies. Science, 305 968-972, (2004)

Park, J.; Y. Jung, P. Kusumah, J., Lee, K. Kwon. Aplication on lonic liquid in Hydrometallurgy, Int. J. Mol. Sci. 1515320 1534, (2014)

Romero, A. Líquidos lónicos a Temperatura Ambiente: Un nuevo medio para las reacciones Químicas. Real Academia de Ciencias Exactas, Físicas y Naturales, 102 (1), 79-90, (2008)

Safamirzaei, M.; H. Modarress. Correlating and predicting low pressure solubility of gases in [bmim][BF4] by neural network molecular modeling. Thermochim. Acta 545 125, (2012)

Sánchez, B.; Prado, L., León, S., González, R. Trabajadores Expuestos a Solventes y Daños a la Salud: una Revisión Sistemática. Revista Colombiana de Salud Ocupacional, 4 (4), 25-29, (2014)

Shamshina, L.; Kelley, S., Gurau, G., Rogers, R. Chemistry: Develop ionic liquid drugs. Nature, doi:10.1038/528188a.https://goo.gl/7pB9gd, acceso: 15 de Agosto de 2017. 528, 188-189, (2015)

Shiflett, M.B.; A. Yokozeki. Chemical absorption of sulfur dioxide in roomtemperature ionic liquids, Ind. Eng. Chem. Res. 49, 1370-1377, (2010)

Tian, S. y otros cuatro autores. Absorption of SO2 at High Temperatures by lonic Liquids and the Absorption Mechanism. Korean Chemical Society, http://dx.doi.org/10.5012/bkcs.2014.35.9.2791, 35 (9), 2791-2796 (2014)

Valderrama, J.; G. Martinez; C.A. Faúndez. Heat capacity of ionic liquids using artificial neural networks and the concept of mass connectivity, Int. J. Thermophys, DOI: https://doi.org/10.1007/s10765-011-0954-6, 32(5), 32:942, (2011)

Valderrama, J. The State of the Cubic Equations of State, Ind. Eng. Chem. Res. 42 (8), 1603-1618, (2003)

Valderrama, J.O.; L.A. Forero, R.E. Rojas. Critical properties and normal boiling temperature of ionic liquids. Update and a new consistency test, Ind. Eng. Chem. Res. 51 7838-7844, (2012)

Valderrama, J.O.; C.A. Faúndez; y J.F. Díaz-Valdes. Equation of state dependency of thermodynamic consistency methods. Application to solubility data of gases in ionic liquids. Fluid Phase Equilibria 449 76-82 (2017a)

Valderrama, J.O.; Campusano, R.A. y Rojas, R.E. Glass transition temperature of ionic liquids using molecular descriptors and artificial neural networks. Comptes Rendus Chimie, doi:10.1016/j.crci.2016.11.009, 20, 573-584, (2017b)

Villada, F., Arroyave, D., y Melissa, V. Pronóstico del precio del petróleo mediante redes neuronales artificiales. Centro de Información Tecnológica. 25(3), 145-154 (2014)

Yokozeki, A.; M.B. Shiflett. Gas solubilities in ionic liquids using a generic van der Waals equation of state, J. Supercrit. Fluids 55 846-851, (2010)

Yokozeki, A.; M.B. Shiflett, Separation of carbon dioxide and sulfur dioxide gases using room-temperature ionic liquid [HMIM][Tf2N], Energy Fuel 23, 4701-4708, (2009) 